\title{
ON THE FIRST HITTING PLACE OF THE INTEGRATED WIENER PROCESS
}

\author{
MARIO LEFEBVRE AND \\ ÉRIC LÉONARD, ${ }^{*}$ École Polytechnique de Montréal
}

\begin{abstract}
Let $d x(t)=y(t) d t$, where $y(t)$ is a one-dimensional Wiener process. In this note, we obtain a formula for the moment-generating function of $y(T)$, where $T$ is the $1 / 2$-winding time about the origin of the integrated Wiener process $x(t)$.
\end{abstract}

\section{Introduction}

Let

$$
\left\{\begin{array}{l}
d x(t)=y(t) d t \\
d y(t)=d W(t)
\end{array}\right.
$$

where $W(t)$ is the standard Wiener process. The two-dimensional process $(x(t), y(t))$ has been studied by McKean (1963), Goldman (1971), Gor'kov (1975) and Lefebvre (1989). Suppose that the process starts at $(x(0), y(0))=(0,1)$ and let

$$
t_{1}=\min \{t: t>0, x(t)=0\} \text {. }
$$

McKean calculated, in particular, the joint distribution of $t_{1}$ and $\left|y\left(t_{1}\right)\right|$, as well as the (marginal) distribution of $\left|y\left(t_{1}\right)\right|$. Next, Goldman gave an expression for the rate of first passage of the integrated Wiener process from $(0, b)$, with $b \leqq 0$, to $x>0$ in terms of the half-winding time of McKean. Gor'kov, for his part, obtained the distribution of $y\left(t_{2}\right)$, where $t_{2}$ is the moment of first passage of the process (1.1) on the half-line $y>0$, starting from $(x, y)$ with $x<0$. Finally, Lefebvre considered the problem of determining the value of $x(t)$ when the Wiener process $y(t)$ hits a barrier in the plane for the first time.

Suppose now that the process $(x(t), y(t))$ starts at $(0, y)$, where $y<0$, and let

$$
T=\min \{t: x(t)=0, y(t) \geqq 0\} .
$$

Next, let $r(y ; v)$ represent the probability density function of $y(T)$; that is,

$$
r(y ; v)=P\{y(T) \in d v \mid y(0)=y\} / d v .
$$

Then, using Gor'kov's result, we may write that

$$
r(y ; v)=\frac{-3^{\frac{3}{2}} v}{4 \pi^{2}} \int_{0}^{\infty} \frac{z^{\frac{3}{2}}}{\left(z^{3}+1\right)\left(z^{2} v^{2}-z v y+y^{2}\right)} d z+\frac{3^{\frac{1}{2}} v}{2 \pi\left(v^{2}+v y+y^{2}\right)}
$$

or, letting $u=-y / v(>0)$,

$$
r(y ; v)=\frac{-3^{\frac{3}{2}}}{4 \pi^{2} v} \int_{0}^{\infty} \frac{z^{\frac{3}{2}}}{\left(z^{3}+1\right)\left(z^{2}+z u+u^{2}\right)} d z+\frac{3^{\frac{1}{2}} v}{2 \pi\left(v^{2}+v y+y^{2}\right)} .
$$

Received 24 April 1989.

* Postal address for both authors: Département de mathématiques appliquées, École Polytechnique de Montréal, Case postale 6079, Succursale "A", Montréal, Québec, Canada H3C 3A7.

Research supported by the Natural Sciences and Engineering Research Council of Canada. 
The integral above may be rewritten as

$$
I=\int_{0}^{\infty}\left[\frac{z^{\frac{1}{2}} A}{z+1}+\frac{z^{\frac{1}{2}} B}{z-w}+\frac{z^{\frac{1}{2}} B^{*}}{z-w^{*}}+\frac{z^{\frac{1}{2}} C}{z+u w}+\frac{z^{\frac{1}{2}} C^{*}}{z+u w^{*}}\right] d z
$$

where the $\operatorname{star}\left({ }^{*}\right)$ denotes the complex conjugate and

$$
\left\{\begin{array}{l}
W=\frac{1}{2}+i 3^{\frac{1}{2}} / 2 \\
A=-\left[3\left(u^{2}-u+1\right)\right]^{-1} \\
B=-i\left[3^{\frac{1}{2}}(w+1)(u+1)\left(w+u w^{*}\right)\right]^{-1} \\
C=-i\left[3^{\frac{1}{2}}(1-u w)(u+1)\left(w^{*}+u w\right)\right]^{-1}
\end{array}\right.
$$

Next, using the fact that

$$
A+B+B^{*}+C+C^{*}=0
$$

we find that

$$
I=-\pi\left\{A+2 \operatorname{Re}\left[B w^{*}\right]+2(u / 3)^{\frac{1}{2}} \operatorname{Re}[C(w+1)]\right\},
$$

which, after some manipulations, may be rewritten as

$$
I=(2 \pi / 3)\left[\frac{u-3^{\frac{1}{2}} u^{\frac{1}{2}}+1}{(u+1)\left(u^{2}-u+1\right)}\right] .
$$

Hence, it follows that

$$
r(y ; v)=\frac{3 v^{\frac{3}{2}}(-y)^{\frac{1}{2}}}{2 \pi\left(v^{3}-y^{3}\right)} .
$$

This formula (with $y=-1$ ) agrees with that of McKean, which he obtained by using the Kontorovich-Lebedev transform. In the next section, we shall apply the same technique as above to obtain the moment-generating function of $y(T)$, which has not been calculated yet.

\section{Moment-generating function of $y(T)$}

Let $M(y ; k)$ denote the moment-generating function of $y(T)$; that is,

$$
M(y ; k)=E\{\exp [-k y(T)] \mid y(0)=y\}=\int_{0}^{\infty} e^{-k v} r(y ; v) d v,
$$

where $k$ is a non-negative constant. Writing $v=-y h$ and $s=-y k$, we find that

$$
M(y ; k)=\frac{3}{2 \pi} \int_{0}^{\infty}\left[A \frac{h^{\frac{1}{2}} e^{-s h}}{h+1}+B \frac{h^{\frac{1}{2}} e^{-s h}}{h-w}+B^{*} \frac{h^{\frac{1}{2}} e^{-s h}}{h-w^{*}}\right] d h,
$$

where

$$
\left\{\begin{array}{l}
w=\frac{1}{2}+i 3^{\frac{1}{2}} / 2\left(=e^{\pi i / 3}\right) \\
A=-\frac{1}{3} \\
B=-i(w+1) 3^{-3 / 2}
\end{array}\right.
$$

Next, if $R$ is a constant which is not a negative real number, we may write that (see Gradshteyn and Ryzhik (1980), p. 319)

$$
\int_{0}^{\infty} \frac{h^{1 / 2} e^{-s h}}{h+R} d h=(\pi / s)^{1 / 2} \exp [s R / 2] D_{-2}\left[(2 s R)^{1 / 2}\right]
$$

for $s>0$, where $D_{-2}(h)$ is a parabolic cylinder function. Furthermore, using the representation of $D_{-2}(h)$ in terms of the error function (see Gradshteyn and Ryzhik, p. 1067), we 
deduce that

$$
\int_{0}^{\infty} \frac{h^{\frac{1}{2}} e^{-s h}}{h+R} d h=(\pi / s)^{\frac{1}{2}}-\pi R^{\frac{1}{2}} e^{s R} \operatorname{erfc}\left[(s R)^{\frac{1}{2}}\right],
$$

where $\operatorname{erfc}(h)$ is the complementary error function. Applying this formula and making use of the fact that

$$
A+B+B^{*}=0
$$

we obtain

$$
\begin{aligned}
M(y ; k)= & e^{s} \operatorname{erfc}\left(s^{\frac{1}{2}}\right) / 2-\frac{i\left(1+w^{*}\right)}{2\left(3^{\frac{1}{2}}\right)} \exp (-s w) \operatorname{erfc}\left(w^{*} s^{\frac{1}{2}}\right) \\
& -\frac{i(1+r)}{2\left(3^{\frac{1}{2}}\right)} \exp \left(-s w^{*}\right) \operatorname{erfc}\left(w s^{\frac{1}{2}}\right) .
\end{aligned}
$$

Now, we have (see Abramowitz and Stegun (1972), p. 297)

$$
\operatorname{erfc}\left(R s^{\frac{1}{2}}\right)=1-2(s / \pi)^{\frac{1}{2}} \sum_{n=0}^{\infty} C(n) R^{2 n+1},
$$

where

$$
C(n)=(-s)^{n}[n !(2 n+1)]^{-1} \text {. }
$$

Hence, since

$$
\operatorname{erfc}(\bar{z})=\overline{\operatorname{erf}(z)}
$$

where $\operatorname{erf}(z)=1-\operatorname{erfc}(z)$ is the error function, we can show the proposition that follows.

Proposition. The moment-generating function of $y(T)$ is given by

$$
\begin{aligned}
M(y ; k)= & e^{s} \operatorname{erfc}\left(s^{\frac{1}{2}}\right) / 2-e^{-s / 2} \cos \left[3^{\frac{1}{2}} s / 2+2 \pi / 3\right] \\
& -2(s / \pi)^{\frac{1}{2}} e^{-s / 2} \sum_{n=0}^{\infty} C(n) \cos \left[3^{\frac{1}{2}} s / 2+2 n \pi / 3\right]
\end{aligned}
$$

where $s=-y k$.

\section{Conclusion}

We have obtained a formula for the moment-generating function of $y(T)$, where $T$ is the 1/2-winding time about the origin of the integrated Wiener process defined by $d x(t)=y(t) d t$. It is easy to verify that $y(T)$ has no finite moments. However, the moment-generating function of $y(T)$ may be needed in some applications. For example, we could use the moment-generating function of $y(T)$, with $T$ defined by

$$
T=\min \{t: x(t)=0, y(t) \geqq 0 \mid x(0)=x \leqq 0, Y(0)=y\}
$$

to obtain the optimal control of the integrated Wiener process.

\section{References}

Abramowitz, M. AND STEgun, I. A. (1972) Handbook of Mathematical Functions with Formulas, Graphs, and Mathematical Tables. Wiley, New York.

Goldman, M. (1971) On the first passage of the integrated Wiener process. Ann. Math. Statist. 42, 2150-2155.

GoR'Kov, JU. P. (1975) A formula for the solution of a boundary value problem for the stationary equation of Brownian motion. Soviet Math. Dokl. 16, 904-908. 
Gradshteyn, I. S. AND Ryzhik, I. M. (1980) Tables of Integrals, Series and Products. Academic Press, New York.

LefebVre, M. (1989) First-passage densities of a two-dimensional process. SIAM J. Appl. Math. 49. MCKeAN, H. P. JR. (1963) A winding problem for a resonator driven by a white noise. J. Math. Kyoto Univ. 2, 227-235. 